\title{
Motility-driven Sperm-sorting Microfluidic Chip with Little Cell Damage for Oligozoospermia Patients
}

\author{
Hong-Yuan Huang, ${ }^{1,2}$ Cheng-Ying Lu, ${ }^{3}$ I-Wen Wang, ${ }^{1}$ and Da-Jeng Yao ${ }^{3,4 *}$ \\ ${ }^{1}$ Department of Obstetrics and Gynecology, Chang Gung Memorial Hospital, \\ No. 5, Fuxing St., Kwei-Shan, Tao-Yuan 333, Taiwan \\ 2 Department of Obstetrics and Gynecology, Chang Gung University and College of Medicine, \\ No. 259, Wen-Hwa 1st Road, Kwei-Shan, Tao-Yuan 333, Taiwan \\ ${ }^{3}$ Department of Power Mechanical Engineering, National Tsing Hua University, \\ No. 101, Sec. 2, Kuang-Fu Rd., Hsinchu 300, Taiwan \\ ${ }^{4}$ Institute of Nanoengineering and Microsystems, National Tsing Hua University, \\ No. 101, Sec. 2, Kuang-Fu Rd., Hsinchu 300, Taiwan
}

(Received September 30, 2019; accepted April 8, 2020)

Keywords: microfluidic chip, sperm sorting, laminar flow, artificial reproduction, oligozoospermia

Various assisted reproductive techniques (ARTs) are now available for infertile couples. Obtaining a large amount of highly active sperm is critical for successful ART operations. Our purpose is to use polydimethylsiloxane (PDMS) microfluid biochip to collect highly active sperm from semen with the characteristic of laminar flow. With a fixed diluted semen viscosity and a fixed flow rate ratio of buffer and diluted semen, this microfluid biochip can sort diluted semen into two groups: sorted and waste. Results of flow cytometry analysis showed that the viability of the sorted group is higher by at least $10 \%$ for both normal and oligozoospermia samples. Our biochip, compared with the traditional centrifuge, is a novel tool for preparing live sperm for ARTs.

\section{Introduction}

The problem of infertility is of increasing concern. The average fertility rate in Taiwan was 1.12 in 2016, implying that each couple has on average 1.12 children. This rate is significantly less than the global average of 2.42 and was the third-lowest birth rate in the world. According to a study, about $15 \%$ of couples are diagnosed as having fertility disorder, which means that they cannot achieve pregnancy normally. About $40 \%$ of these couples with fertility disorder result from male problems and $10 \%$ arise from both the male and female having problems. In this work, we focused on infertility problems that are mostly related to abnormal morphology, poor mobility, or small quantity of sperm. Our research is thus dedicated to the acquisition of highquality sperm for medical applications.

In the current technology of reproductive medicine, an assisted reproductive technique $(\mathrm{ART})^{(1-5)}$ can improve fertility through artificial treatments, ${ }^{(6-8)}$ including in vitro fertilization (IVF) and intracytoplasmic sperm injection (ICSI). The purpose of ART is to fertilize the best oocyte with the best sperm; ${ }^{(9)}$ sorting the sperm is thus extremely important. Traditional ${ }^{*}$ Corresponding author: e-mail: djyao@mx.nthu.edu.tw https://doi.org/10.18494/SAM.2020.2643 
sperm sorting methods include the swim-up method, density gradient centrifugation, and centrifugation-sperm washing. However, these methods suffer from disadvantages, such as a large centrifugal force and a long process time. These disadvantages might impair the sperm quality or lead to sperm separation, resulting in a failed fertilization. ${ }^{(10,11)}$

Among microfluidic systems, the efficiency of sorting live sperm has been enhanced with a biomedical microfluidic chip to avoid sperm damage, and only a few samples must be analyzed. ${ }^{(12-15)}$ We applied various sperm samples to a microfluidic chip with a novel sorting mechanism in our previous work; ${ }^{(16)}$ this method can achieve the inexpensive and efficient separation of sperm cells for use in artificial reproduction. It was confirmed in previous studies that the distribution of laminar flow formed by syringe pumps and a microchannel with three inlets and three outlets can isolate about 10000 motile sperm cells and increase their viability. ${ }^{(16-18)}$ The aim of this research was to apply the present invention to semen samples of various concentrations and viscosities so as to further improve the viability and quality of sperm from both normal persons and oligozoospermic patients.

\section{Materials and Methods}

We previously proposed a microfluidic chip system based on laminar flow to select live sperm, ${ }^{(19,20)}$ which combined a design with three inlets and three outlets ${ }^{(21)}$ and a main diffusion channel. ${ }^{(22)}$ Figure 1 shows the schematic design. The main separation channel was designed to have a diffusor structure from the inlet to the outlet. According to the flow rate equation $Q$ $=A \times V$, where $Q$ is the flow rate; $A$ is the area; and $V$ is the flow velocity, increasing the crosssectional area will decrease the flow rate, increasing the likelihood that sperm cells are sorted. We used a syringe pump to infuse the semen and the buffer from the inlets and generate twophase flow. The fluids are not mixed together because of the laminar flow, which can separate motile sperm from dead sperm on the basis of their own motility.

A computed-aided simulation software (ANSYS) simulation was used to analyze the distribution of semen for our input velocities in the microchannel, enabling us to determine the appropriate experimental flow rates to sort semen. The semen viscosity $(\mu)$ was mostly

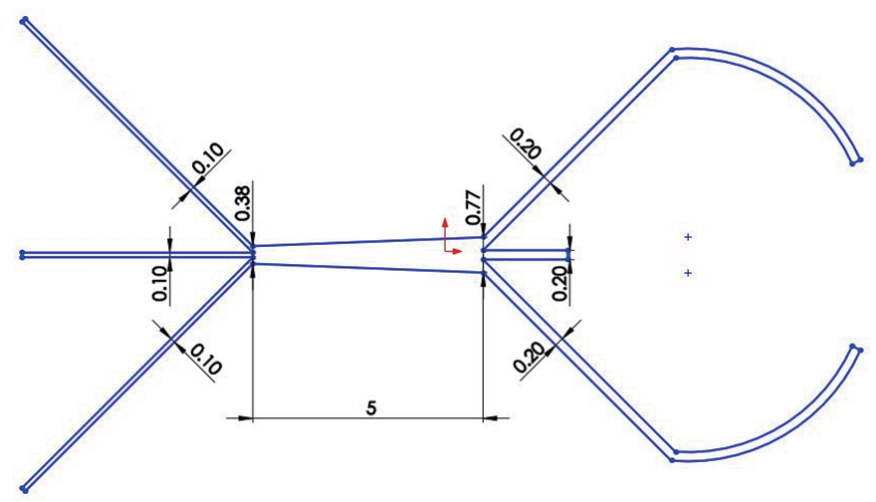

Fig. 1. (Color online) Schematic of the microchannel (widths of inlets, $100 \mu \mathrm{m}$; widths of outlets, $200 \mu \mathrm{m}$ ). The width of the main diffusion channel was increased from 383 to $766 \mu \mathrm{m}$, and the height is $100 \mu \mathrm{m}$. The semen is infused from the side inlet and the buffer is injected into the central inlet. The outlet in the middle is the sorting outlet with waste outlets on the sides. 
in the range of 1-6 mPass, and the ratio of the infused flow rate between the two inlets should be adjusted according to the variable viscosity. As shown in Fig. 2, when $\mu$ was $2 \mathrm{mPa}$.s and the ratio of buffer and semen flow rates was $2: 1$ or $4: 1$, the semen (red part) entered the central sorting outlet, which decreased the sorting efficiency. Furthermore, when we used a flow rate ratio of $8: 1$ with the semen viscosity of $2 \mathrm{mPa} \cdot \mathrm{s}$, the sperm flowed into the waste outlet. Flow rate ratios of 11:1-20:1 produced similar results for semen viscosities of 3-6 $\mathrm{mPa} \cdot \mathrm{s}$.

After sorting, flow cytometry with SyBr14 and PI fluorescence was used to analyze the quantity and viability of the sperm, and we investigated the efficiency of sorting on the basis of the obtained data. The fabrication of the chip involved a thick film of SU8, a negative photoresist, ${ }^{(23)}$ and polydimethylsiloxane (PDMS) soft lithography, combined with glass to form the microchannel. Figure 3 shows the fabrication and actual picture of the biochip.

Photolithography with the SU8-3050 negative photoresist was used to fabricate the mother mold of the microchannel structure. UV exposure effectively transferred the pattern to the mother mold to produce a microscale structure. The photoresist was spin-coated at $500 \mathrm{rpm}$ for $10 \mathrm{~s}$ and $1300 \mathrm{rpm}$ for $30 \mathrm{~s}$ to attain a height of $100 \mu \mathrm{m}$. During the UV exposure, the gap

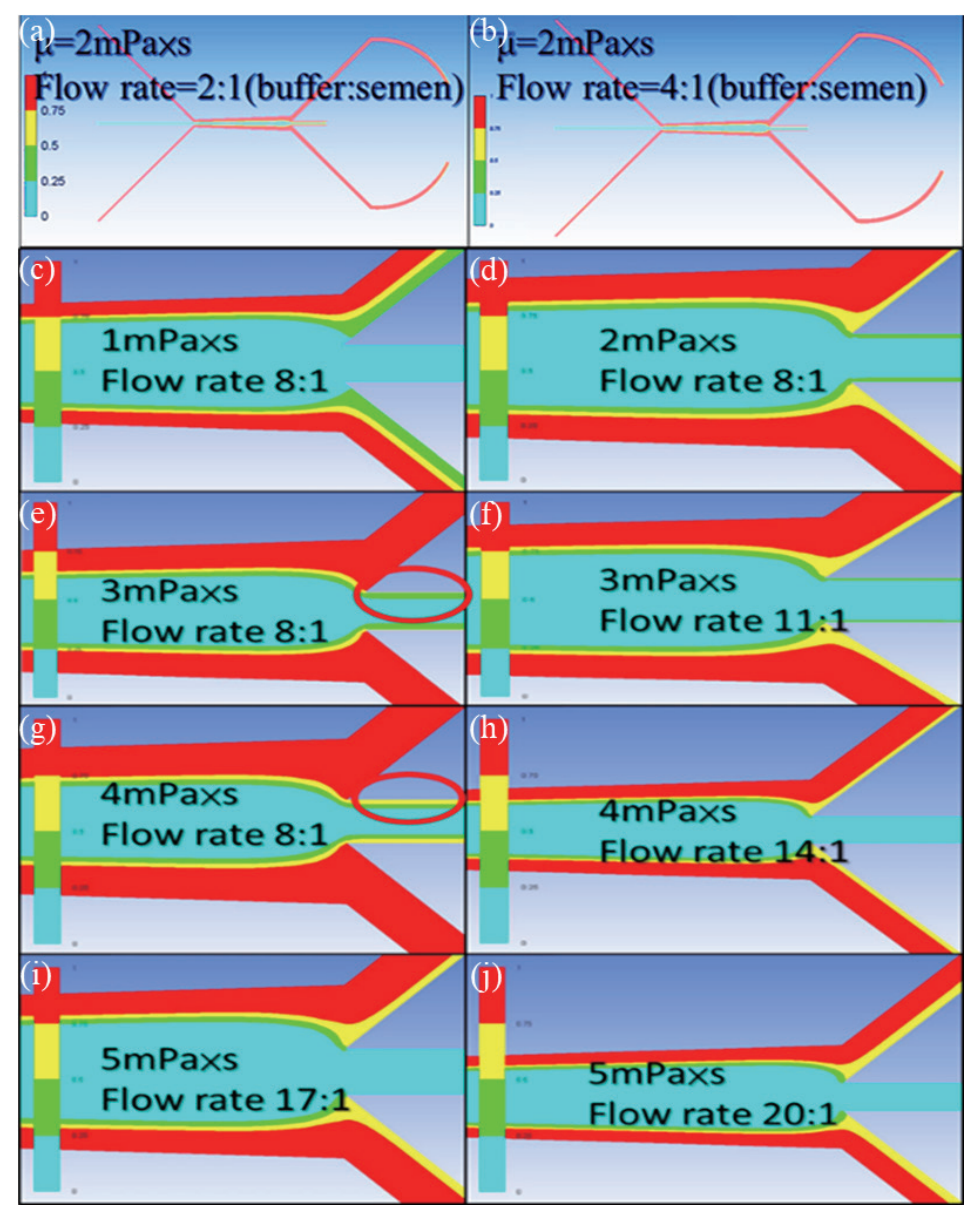

Fig. 2. (Color online) Distribution of semen in the microchannel according to an ANSYS simulation. At flow rate ratios of 2:1 and 4:1 (buffer: semen), the semen (red part, 75-100\%) enters the sorting outlet. The optimal flow rate ratio for sorting is 8:1-20:1 depending on the viscosity of the semen. 


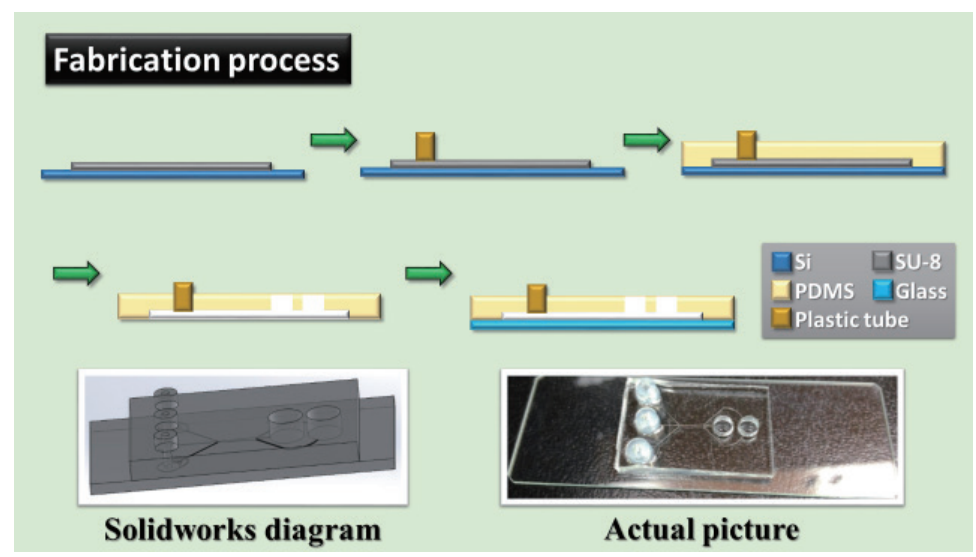

Fig. 3. (Color online) Fabrication of the sperm-sorting chip. The pattern of the microchannel is formed on a $\mathrm{Si}$ wafer with a SU8-3050 negative photoresist and printed on a PDMS substrate. The PDMS substrate and glass slide are bonded together with $\mathrm{O}_{2}$ plasma to complete the process.

between the silicon wafer and the mask was minimized to avoid UV light diffraction and the generation of a standing wave, which might damage the structural integrity of the horizontal and vertical flatness of the film.

After the fabrication of the mother mold, the pattern was transferred to PDMS by soft lithography. ${ }^{(24)}$ In this process, a plastic tube (inner diameter, $3 \mathrm{~mm}$; height, $1 \mathrm{~mm}$ ) was placed on each inlet, which served to connect the chip and the syringe. We then poked holes into the outlets to form sorting and waste reservoirs after curing the PDMS (A:B $=20 \mathrm{~g}: 2 \mathrm{~g}$ ).

The patterned PDMS was finally bonded to a glass slide. An electrostatic generator generated an $\mathrm{O}_{2}$ plasma to oxidize the surface of the PDMS, turning the hydrophobic $-\mathrm{O}-\mathrm{Si}\left(\mathrm{CH}_{3}\right)_{2}-$ bond into a hydrophilic $-\mathrm{O}_{n} \mathrm{Si}(\mathrm{OH})_{4-n}-$ bond. ${ }^{(25)}$ After the surface modification, we attached the oxidized PDMS to the glass slide to complete the fabrication of the microchannel.

\section{Experimental Process}

\subsection{Stock sample sorting}

Viscosity plays an important role in the sorting process: using an inappropriate flow rate leads to failure of the experiment. We first tried to perform simulations using ANSYS. The results shown in Fig. 2 were obtained by using the following parameters: $\mu=1-2 \mathrm{mPa} \cdot \mathrm{s}$ for a flow rate ratio of 0.4:0.05 $\mu \mathrm{l} / \mathrm{min}$ (buffer:semen), $\mu=2-3 \mathrm{mPa} \cdot \mathrm{s}$ for a flow rate ratio of 0.55:0.05 $\mu \mathrm{l} / \mathrm{min}, \mu=3-4 \mathrm{mPa} \cdot \mathrm{s}$ for a flow rate ratio of 0.7:0.05 $\mu 1 / \mathrm{min}, \mu=4-5 \mathrm{mPa} \cdot \mathrm{s}$ for a flow rate ratio of $0.85: 0.05 \mu \mathrm{l} / \mathrm{min}$, and $\mu=5-6 \mathrm{mPa} \cdot \mathrm{s}$ for a flow rate ratio of $1: 0.05 \mu \mathrm{l} / \mathrm{min}$.

To obtain the viscosity in the experiments, we measured the semen viscosity (Sekonic Viscomate, VM-10A-L) before sorting and infused bovine serum albumin (BSA, 1\%) solution into the microchannel to provide a hydrophilic coating at the same time. The first step in the sorting process was to push the syringes and ensure, by microscopic observation, that the 
semen and buffer had already reached the microchannel. Second, the semen flow rate was set to $0.05 \mu \mathrm{l} / \mathrm{min}$; the flow rate of the buffer used to clean the microchannel was set to $50 \mu 1 / \mathrm{min}$. Third, the buffer flow rate was set to $0.4-1 \mu \mathrm{l} / \mathrm{min}$ with sorting for $1 \mathrm{~h}$. The sample was finally dyed with $\operatorname{SyBr} 14(0.5 \mu \mathrm{l} / \mathrm{ml})$ and PI $(5 \mu 1 / \mathrm{ml}),{ }^{(26)}$ and analyzed with a flow cytometer.

\subsection{Sorting at fixed viscosity}

Motile sperm cells in a stock sample were separated at various flow rates, but large velocity ratios (11:1-20:1) prevented the motile sperm cells from swimming through the boundaries of the fluids, which decreased the sorting efficiency. Hence, we diluted the semen to obtain a fixed viscosity of $\mu=2 \mathrm{mPa} \cdot \mathrm{s}$ and carried out sorting at a fixed flow rate ratio of $8: 1$. With the fixed viscosity of $2 \mathrm{mPa} \cdot \mathrm{s}$, more live sperm cells were obtained at the low flow ratio than at the high flow ratio.

\subsection{Sorting concentration of oligozoospermia}

The goals of this research were to help infertile patients who acquire live sperm and to implement ARTs. One type of male infertility is oligozoospermia, which corresponds to a sperm concentration of $10 \times 10^{6}-15 \times 10^{6} \mathrm{sperm} / \mathrm{ml}$. Moderate oligozoospermia corresponds to a sperm concentration of $5.0 \times 10^{6}-10 \times 10^{6} \mathrm{sperm} / \mathrm{ml}$, and severe oligozoospermia corresponds to a sperm concentration of less than $5.0 \times 10^{6} \mathrm{sperm} / \mathrm{ml}$.

Because of the rarity of the sperm in oligozoospermia samples, we imitated the sorting of semen in a sample with a low sperm concentration through dilution. The concentrations of sperm before and after dilution were determined with a counting chamber (Makler). The diluted oligozoospermia sample was sorted at a flow rate ratio of 8:1 for $1 \mathrm{~h}$ and analyzed with a flow cytometer.

\subsection{Detection of integrity of cell membrane}

Because the PI dye cannot permeate a cell membrane, it can only dye dead cells with broken cell membranes. We applied this phenomenon to determine whether a cell membrane was intact or not and compared the result of a microfluidic group with that of a centrifugal group. The samples used in the centrifugal process were prepared by adding $30 \mu \mathrm{l}$ of stock semen to $1970 \mu \mathrm{l}$ of buffer, then centrifugation was carried out at $1800 \mathrm{rpm}$ for $15 \mathrm{~min}, 1000 \mathrm{rpm}$ for $10 \mathrm{~min}$, and $1000 \mathrm{rpm}$ for $10 \mathrm{~min}$. The control, microfluidic, and centrifugal groups were then dyed with SyBr14 and PI, and analyzed with a flow cytometer.

\section{Results}

A flow cytometer can identify the type and strength of fluorescence, and categorize the cells, ${ }^{(27)}$ as shown in Fig. 4. This property is helpful for analyzing the sorting results, such as for the two parameters defined below. 


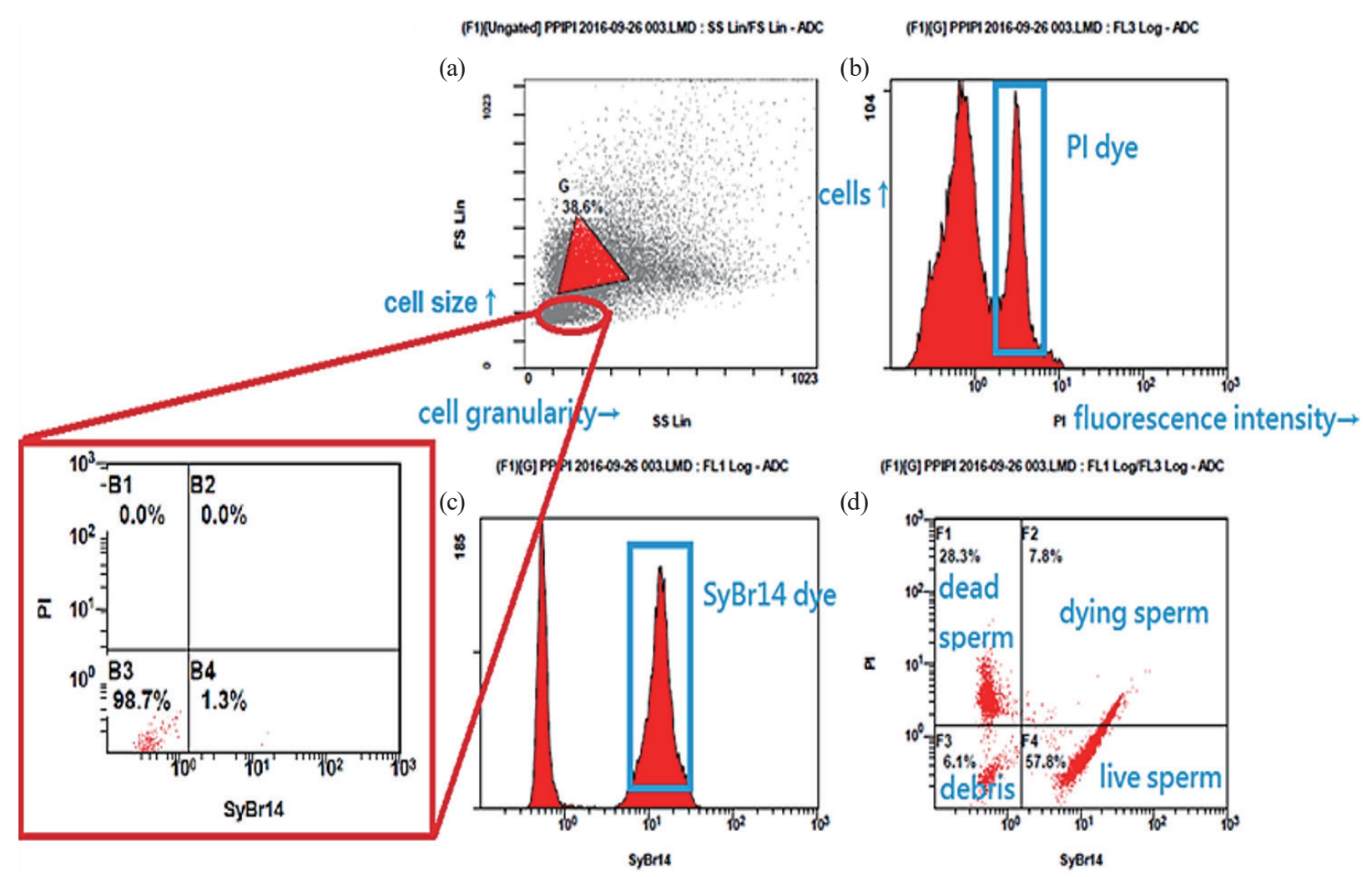

Fig. 4. (Color online) Flow cytometry result. (a) The machine detects the cell size ( $y$-axis) and granularity ( $x$-axis) using forward- and side-scattered laser light. (b) Detection of PI, excited fluorescence strength. (c) Detection of SyBr14, excited fluorescence strength. (d) Dot map obtained from fluorescence signals.

In reference to the four-quadrant diagram in Fig. 4, the semen viability is defined as

$$
\text { viability }=\frac{\text { livesperm }(\mathrm{F} 4)}{\text { livesperm }(\mathrm{F} 4)+\text { dying } \operatorname{sperm}(\mathrm{F} 2)+\text { dead } \operatorname{sperm}(\mathrm{F} 1)} \times 100 \% \text {. }
$$

Dying sperm cells emit both green and red fluorescence, which means that the cell membranes are broken and cannot be used in ARTs. The F3 region has neither green nor red fluorescence detected. This region corresponds to debris and can be neglected.

The sorting efficiency is defined as sorting efficiency $=\frac{\text { live sperms in sorting outlet }(\mathrm{F} 4)}{\text { live sperms in sorting outlet }(\mathrm{F} 4)+\text { live sperms in waste outlet }(\mathrm{F} 4)} \times 100 \%$. (2)

A higher sorting efficiency indicates more live sperm cells were sorted from infused live sperm cells. 


\subsection{Stock sample sorting}

From the results of the ANSY simulation, we knew that a flow rate ratio of $8: 1$ is inapplicable to semen of high viscosity (greater than $2 \mathrm{mPa} \cdot \mathrm{s}$ ) because semen enters the sorting outlet. However, the flow rate ratios can be adjusted for highly viscous semen, and this method is effective. The difference in flow field with flow rate ratios of 8:1 and 17:1 is shown in Fig. 5; less than $25 \%$ of the semen (blue part) flowed into the sorting outlet with the flow rate ratio of 17:1.

After many experiments with different flow rates, we observed by microscopy that most sperm cells enter the waste outlet, which is expected to improve the separation. According to Table 1, the viability was significantly improved with the fixed flow rate ratio of $8: 1$ in the four

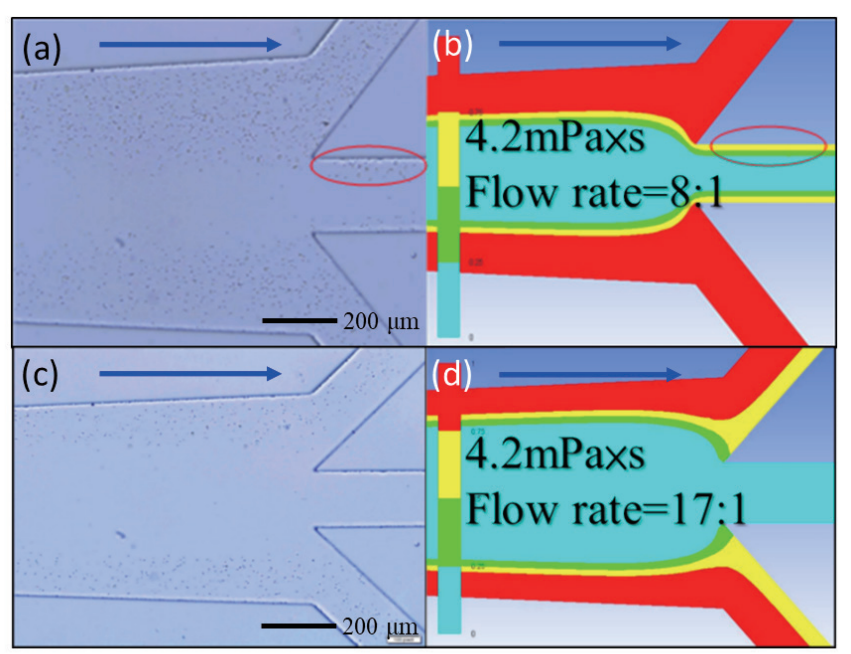

$\longrightarrow$ Direction of flow $\bigcirc$ Semen rush into the sorting outlet

Fig. 5. (Color online) Highly viscous semen sorted at flow rate ratios of $8: 1$ and 17:1. The viscosity of $4.2 \mathrm{mPa} s$ and the flow rate ratio of $8: 1$ result in $50-75 \%$ (yellow part) of the semen entering the sorting outlet, but less than $25 \%$ of the semen enter the sorting outlet when the ratio is increased to $17: 1$. This adjustment improves the separation of a stock sample in the biochip.

Table 1

Results of stock sample sorting before and after adjustment of the flow rate. Before: all viability enhancements were less than $10 \%$ (gray columns). After: the viability enhancements were greatly improved, but the sorting efficiencies were very low (white columns).

\begin{tabular}{lcccccc}
\hline Sample & $\begin{array}{c}\text { Flow rate ratio } \\
\text { buffer:semen }\end{array}$ & $\begin{array}{c}\text { Viscosity } \\
(\mathrm{mPa} \cdot \mathrm{s})\end{array}$ & $\begin{array}{c}\text { Control } \\
\text { viability (\%) }\end{array}$ & $\begin{array}{c}\text { Sorting } \\
\text { viability (\%) }\end{array}$ & $\begin{array}{c}\text { Viability } \\
\text { enhancement (\%) }\end{array}$ & $\begin{array}{c}\text { Sorting } \\
\text { efficiency (\%) }\end{array}$ \\
\hline A & & 2.1 & 37.1 & 44.8 & 20.8 & 37.9 \\
B & $8: 1$ & 2.95 & 55 & 52.8 & -4.0 & 25.9 \\
C & & 4.2 & 61.5 & 62 & 0.8 & 5.3 \\
D & & 5.1 & 58.5 & 65.8 & 12.5 & 3 \\
\hline A & $11: 1$ & 2.1 & 30.6 & 61.6 & 101.3 & 8.9 \\
B & $14: 1$ & 2.95 & 55 & 61.8 & 12.4 & 2.2 \\
C & $17: 1$ & 4.2 & 61.5 & 86.2 & 40.2 & 0.5 \\
D & $20: 1$ & 5.1 & 58.5 & 78 & 33.3 & 0.2 \\
\hline
\end{tabular}


groups of experiments. This result confirms that our biochip can sort live sperm from a stock sample with an appropriate flow rate, but increasing the flow rate of the buffer prevented many live sperm cells from swimming through the interface of the fluids and flowing into the waste tank. As a result, even at a large initial concentration, the sorting efficiency was only $0.2 \%$ and not enough live sperm cells were collected.

\subsection{Fixed-viscosity sorting}

To solve the problem of sorting the stock sample, we diluted the semen to obtain a viscosity of $\mu=2 \mathrm{mPa} \cdot \mathrm{s}$ and carried out many experiments. We took the averages of the results and compared them with the result of stock sample sorting. According to Table 2, the average concentration of the stock samples was $34 \times 10^{6} / \mathrm{ml}$ and that of the diluted samples was $19.1 \times 10^{6} / \mathrm{ml}$. The average motility $(\mathrm{a}+\mathrm{b})$ of the stock samples was $49.7 \%$ and that of the diluted samples was $44.3 \%$, which does not show a large difference. The mobility of sperm is classified as follows:

a: rapid forward movement, no tail movement visible,

b: wiggling left and right, tail movement visible,

c: wiggling in a fixed place,

d: no movement.

The viabilities were 53.1 and $57.2 \%$ before sorting, 73.1 and $74.6 \%$ after sorting, and the increases were 20 and $17.4 \%$. These results show that the viability enhancements of the stock sample with a high ratio and the diluted sample with a ratio of $8: 1$ are similar; both can increase the number of live sperm cells by about $20 \%$, but there is a major difference between these two methods in terms of sorting efficiency. Although the sperm concentration of the stock sample is large, only $2 \%$ of the live sperm (about 2098 live sperm on average) were separated because of the high flow rate. In contrast, although the samples diluted to a viscosity of $2 \mathrm{mPa} \cdot \mathrm{s}$ had a low sperm concentration, approximately 10\% of live sperm were sorted (about 7819 live sperm on average). These results indicate that, under similar viability enhancements, sorting a diluted sample with a flow rate ratio of 8:1 facilitates the filtering out the live sperm, making it a superior sorting method.

Table 2

Comparison between stock sample sorting and fixed-viscosity sorting. The viability and viability enhancement are similar, but the sorting efficiency and the number of acquired live sperm cells after sorting a diluted sample were much greater for the fixed-viscosity sorting.

\begin{tabular}{lcc}
\hline & $\begin{array}{c}\text { Stock sample with } \\
\text { high flow rate ratio }(N=5)\end{array}$ & $\begin{array}{c}2 \mathrm{mPa} \cdot \mathrm{s} \text { diluted sample with } \\
8: 1 \text { flow rate ratio }(N=16)\end{array}$ \\
\hline Concentration (ml) & $34 \times 10^{6}$ & $19.1 \times 10^{6}$ (diluted) \\
Motility a + b (\%) & 49.7 & 44.3 \\
Control viability (\%) & 53.1 & 57.2 \\
Sorting viability (\%) & 73.1 & 74.6 \\
Viability enhancement (\%) & 20 & 17.4 \\
Sorting efficiency (\%) & 2 & 9.5 \\
Sorting live sperms & 2098 & 7819 \\
\hline
\end{tabular}




\subsection{Sorting results by different concentration of oligozoospermia patients}

As defined by WHO in 2010, ${ }^{(28)}$ there are three levels of oligozoospermia according to the sperm concentration. The samples compared with different levels of oligozoospermia were obtained by dilution, and the sorting results of diluted samples with different levels of oligozoospermia are shown in Fig. 6.

First, we diluted semen to a mild oligozoospermia concentration, carried out many experiments, and compared the average viability enhancements. As shown in the left of Fig. 6, the average proportions of live sperm in the mild oligozoospermia sample were about 69.8 and $84.4 \%$ before and after sorting, respectively, with an average enhancement of $21 \%$ and a sorting efficiency of $14.7 \%$.

Second, as shown at the center of Fig. 6, the viability of the moderately diluted oligozoospermia samples was increased from 68.5 to $81.5 \%$ after sorting, with an average enhancement of $19.0 \%$ and a sorting efficiency of $13.8 \%$.

Third, as shown in the right of Fig. 6, the viability of the severely diluted oligozoospermia samples was increased from 67.6 to $86.4 \%$ after sorting, with an average enhancement of $27.9 \%$ and a sorting efficiency of $13.7 \%$.

These results indicate that, at each level of oligozoospermia, the viabilities and sorting efficiencies were similar. A comparison of the results with those for the sorting of normal semen shows that the sorting performance for an oligozoospermia sample is comparable to that for a normal sample, which means that the biochip is applicable to all semen concentrations.

\subsection{Detection of integrity of cell membrane}

The average results of several experiments in Fig. 7 show that the percentage of sperm cells with an intact membrane from the waste outlet was similar to that of the control group

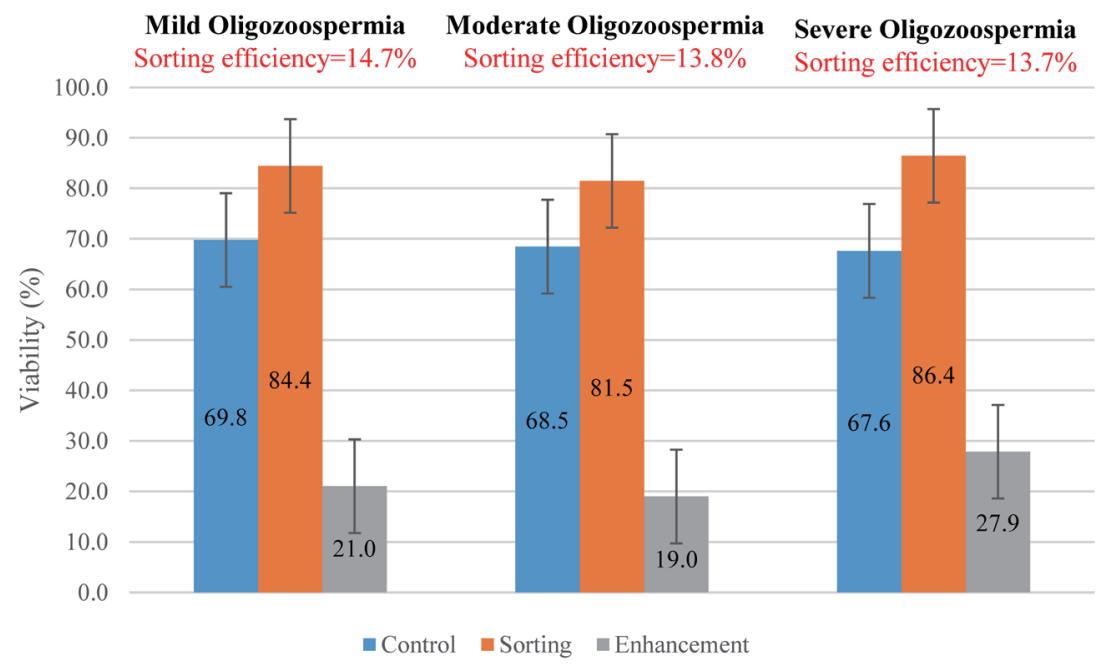

Fig. 6. (Color online) Results of sorting diluted oligozoospermia samples. The viability enhancements and sorting efficiencies of these samples with three levels of oligozoospermia are similar, which means that the biochip is applicable to all semen concentrations. 


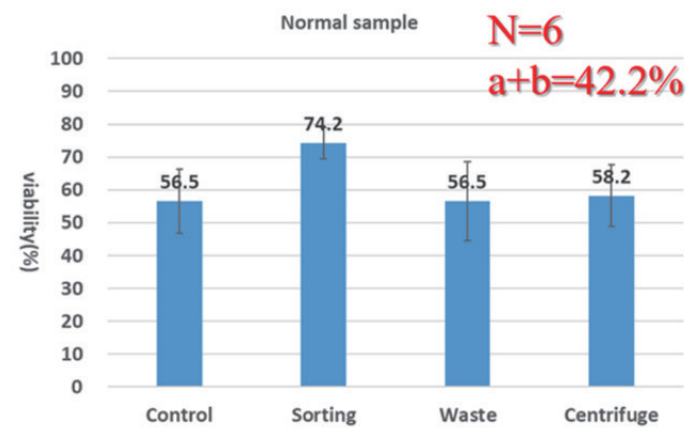

(a)

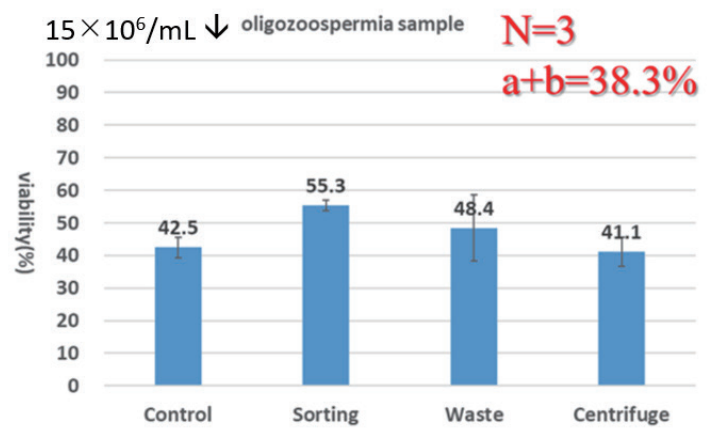

(b)

Fig. 7. (Color online) Results of detection of cell membrane integrity. In the two types of samples, the integrity of the sorted group is $10-20 \%$ greater than those of the other groups, showing that the fraction of intact sperm cells is improved after sorting in the biochip.

because the semen separated out only some of the live sperm cells into the sorting outlet. In the centrifugation group, all sperm cells were collected and precipitated; thus, the proportion of sperm cells with an intact membrane was also similar to that of the control group, but the proportion of sperm cells with an intact membrane in the sorting outlet was about 10-20\% greater than those for the other groups because of the removal of dead sperm cells during the sorting, confirming that the sperm integrity after sorting was superior.

We divided the experimental results into two parts according to the sperm concentration: normal sample (more than $\left.15 \times 10^{6} / \mathrm{ml}\right)$ and oligozoospermia $\left(15 \times 10^{6} / \mathrm{ml}\right.$ or less). The results of the control group, the waste outlet, and the centrifuge group were similar, whereas the sorting outlet had 74.2 and $55.3 \%$ intact sperm cells for the normal and oligozoospermia samples, respectively. Moreover, the results of the oligozoospermia sample before and after sorting were inferior to those of the normal sample; we infer that patients with oligozoospermia have a greater probability of suffering asthenospermia and decreased viability.

\section{Conclusions}

In this study, a syringe pump with a low flow rate was used in combination with microfluidic chips. Laminar flow was generated on a microscale and applied to sperm samples with various concentrations and motilities. This method can replace traditional screening methods and manual selection, and also avoid possible harm to sperm during centrifugation.

The biochip used in stock sample sorting can still give excellent screening results. Although the viscosities of the stock semen were mostly high, these high viscosities altered the distribution of the flow field in the microchannel. Dead sperm cells could not be removed effectively, affecting the viability of the sorting outlet. After the appropriate adjustment of the flow rate ratio (11:1-20:1), the viability was greatly improved, even when the viscosity was less than $2 \mathrm{mPa} \cdot \mathrm{s}$ or greater than $5 \mathrm{mPa} \cdot \mathrm{s}$. Although the quantity of sorted live sperm cells and the sorting efficiency were low, it proved that both diluted and stock samples can be used with this chip.

To improve the efficiency of sorting a stock sample and to facilitate operation by users, the samples were diluted to obtain a fixed viscosity of $2 \mathrm{mPa} \cdot \mathrm{s}$. Then, all the samples were 
uniformly sorted with a flow rate ratio of 8:1 (buffer:semen). According to the experimental results, although the average viability of the oligozoospermia samples was low, the viabilities of both normal and oligozoospermia samples were increased by more than $10 \%$ with a sorting efficiency of about $10 \%$. Provided that the sperm motility was sufficient, all samples were successfully screened in this chip.

The experiment on sorting diluted oligozoospermia samples showed that the average viability was increased by at least $10 \%$ after the screening by the chip, regardless of the sample concentration. The proportion of live sperm cells screened out (sorting efficiency) was more than $10 \%$, confirming that the chip enhanced the sperm viability and had the ability to collect live sperm cells at all concentrations.

The experiment on the integrity of the cell membrane showed that the damage rates of sperm cells screened with the microchannel decreased by to 10-20\%, which means that live sperm cells were screened with little damage.

\section{Acknowledgments}

The authors are grateful for the financial support from the Taiwan Ministry of Science and Technology (MOST 106-3114-B-182A-002-; MOST 107-2321-B-182A-004-, MOST 107-2622-E-007-006-CC2, and MOST 104-2221-E-007-078-MY2) and CGMH funds (CMRPG3E0771; CMRPG3G1362; CMRPG3H1661). Chang Gung Memorial Hospital in Taiwan supplied human semen samples and observation equipment (IRB\#: 104-7808B/201700425B0). The materials and equipment used in the fabrication process were provided by the laboratory of Prof. Fan-Gang Tseng and Prof. Cheng-Hsien Liu at National Tsing Hua University, Taiwan.

\section{References}

1 R. Edwards: Reprod. Biomed Online 7 (2003) 506.

2 R. Dickey: Fertil Steril. 88 (2007) 1554.

3 V. Allen, R. Wilson, and A. Cheung: J. Obstet Gynaecol Can. 28 (2006) 220.

4 P. Setti, M. Cavagna, E. Albani, G. Morreale, P. Novara, A. Cesana, and V. Parini: Reprod. Biomed. Online 11 (2005) 64.

5 K. Patricia, N. Robert, and S. Jonathan: Nat. Med. 8 (2002) S29.

6 R. R. Henkel and W.-B. Schill: Reprod. Biol. Endocrinol. 1 (2003) 22.

7 S. Gordts, R. Campo, P. Puttemans, I. Brosens, M. Valkenburg, J. Norre, M. Renier, D. Coeman, and S. Gordts: Reprod. Biomed Online 10 (2005) 436.

8 Z. Pandian, S. Bhattacharya, O. Ozturk, G. Serour, and A. Templeton: Cochrane Database Syst Rev. 18 (2004) CD003416.

9 F. Zegers-Hochschild, G. D. Adamson, J. de Mouzon, O. Ishihara, R. Mansour, K. Nygren, E. Sullivan, and S. Van der Poel: Human Reprod. 24 (2009) 2683.

10 A. Zini, A. Finelli, D. Phang, and K. Jarvi: Urology 56 (2000) 1081.

11 V. Jayaraman, D. Upadhya, P. K. Narayan, and S. K. Adiga: J. Assist. Reprod. Genet. 29 (2012) 557.

12 Y.-A. Chen, Z.-W. Huang, F.-S. Tsai, C.-Y. Chen, C.-M. Lin, and A. Wo: Microfluid. Nanofluid. 10 (2011) 59.

13 M. Yamada, K. Kano, Y. Tsuda, J. Kobayashi, M. Yamato, M. Seki, and T. Okano: Biomed. Microdevices 9 (2007) 637.

14 C.-H. Lin, C.-Y. Lee, C.-H. Tsai, and L.-M. Fu: Microfluid. Nanofluid. 7 (2009) 499.

15 H.-Y. Huang, Y.-L. Lai, and D.-J. Yao: Micromachines 9 (2018) 135.

16 H.-Y. Huang, P.-W. Huang, and D.-J. Yao: Microsyst. Technol. 23 (2017) 305.

17 Y.-H. Chung, Y.-H. Hsiao, W.-L. Kao, C.-H. Hsu, D.-J. Yao, and C. Chen: Biomicrofluidics 9 (2015) 022407.

18 H.-Y. Huang, D.-J. Yao, T.-L. Wu, H.-R. Huang, C.-J. Li, H.-T. Fu, Y.-K. Soong, and M.-Y. Lee: J. Lab. Autom. 19 (2014) 91. 
19 K. Matsuura, M. Takenami, Y. Kuroda, T. Hyakutake, S. Yanase, and K. Naruse: Reprod. Biomed. Online 24 (2012) 109.

20 B. S. Cho, T. G. Schuster, X. Zhu, D. Chang, G. D. Smith, and S. Takayama: Anal. Chem. 75 (2003) 1671.

21 S. Koyama, D. Amarie, H. A. Soini, M. V. Novotny, and S. C. Jacobson: Anal. Chem. 78 (2006) 3354.

22 Y. N. Lin, P. C. Chen, R. G. Wu, L. C. Pan, and F. G. Tseng: Proc. 2013 IEEE 26th Int. Conf. (IEEE, 2013) $1153-1156$.

23 C.-H. Chen, S.-C. Chuang, H.-C. Su, W.-L. Hsu, T.-R. Yew, Y.-C. Chang, S.-R. Yeh, and D.-J. Yao: Lab Chip 11 (2011) 1647.

24 D. A. Chang-Yen, R. K. Eich, and B. K. Gale: J. Lightwave Technol. 23 (2005) 2088.

25 B. E. Slentz, N. A. Penner, and F. E. Regnier: J. Chromatogr. A 948 (2002) 225.

26 D. L. Garner and L. A. Johnson: Biol. Reprod. 53 (1995) 276.

27 S. C. Chu, T. F. Wang, C. C. Li, R. H. Kao, D. K. Li, Y. C. Su, D. A. Wells, and M. R. Loken: Leukemia Res. 35 (2011) 868.

28 World Health Organization: WHO Laboratory Manual for the Examination and Processing of Human Semen (2010).

\section{About the Authors}

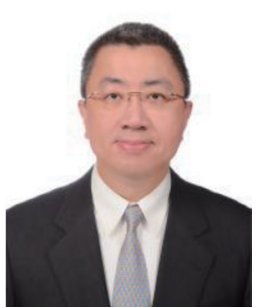

Hong-Yuan Huang received his MD degree from Chung-Shan Medical University, Taiwan, in 1987. He was appointed as Professor of Obstetrics and Gynecology at Chang Gung Memorial Hospital, Taiwan. His research is aimed at various aspects of clinical and reproductive medicine, especially in reproductive immunology, endometriosis, and nanomedicine.

(hongyuan@cgmh.org.tw)

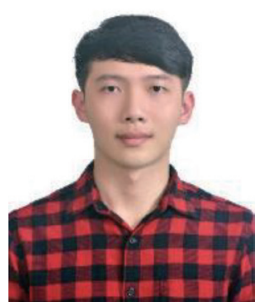

Cheng-Ying Lu received his B.S. degree from National Tsing Hua University (NTHU), Taiwan, in 2015 and his M.S. degree from the Department of Power Mechanical Engineering, NTHU, Taiwan, in 2018. After graduation, he joined KYMCO as an engineer. His research interests are in MEMS, bioengineering, and microfluidics. (j5549001@yahoo.com.tw)

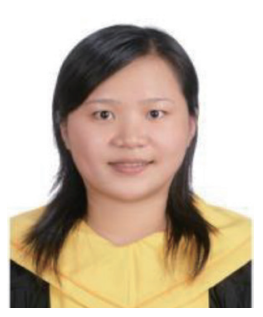

I-Wen Wang received her B.S. degree from National Chiayi University, Taiwan, in 2008. Since 2010, she has been a research associate at Chang Gung Memorial Hospital, Taiwan. Her research interests are in molecular biology and nanomedicine. (iwen0711@gmail.com)

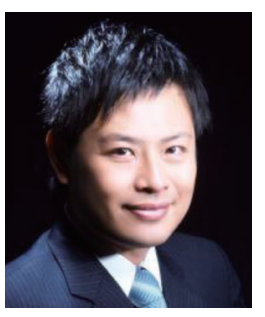

Da-Jeng Yao is a professor at the Institute of NanoEngineering and MicroSystems (NEMS), National Tsing Hua University, Taiwan. He received his MS degree from the Department of Mechanical Engineering, Lehigh University in 1996, and his Ph.D. degree from the Department of Mechanical and Aerospace Engineering, University of California, Los Angeles (UCLA) in 2001. His research interests are in fertilization on a chip, intelligent gas sensing systems and their applications, digital microfluidic systems, and $\mathrm{THz}$ applications. (djyao@mx.nthu.edu.tw) 